BULLETIN OF THE

AMERICAN MATHEMATICAL SOCIETY

Volume 79, Number 2, March 1973

\title{
PROJECTIVE MODULES FOR FINITE CHEVALLEY GROUPS
}

\author{
BY J. E. HUMPHREYS ${ }^{1}$ AND D. N. VERMA
}

Communicated by George B. Seligman, September 12, 1972

1. Introduction. The irreducible modular representations of the finite Chevalley groups (and their twisted analogues) have been described by $C$. W. Curtis and R. Steinberg (see [1], [2], [8]). In this note we outline some parallel results on principal indecomposable modules (PIM's), for which proofs will appear elsewhere. The groups $\mathbf{S L}(2, q)$ are already treated in detail in [5], based in part on the method of A.V. Jeyakumar [6].

$K$ denotes an algebraically closed field of prime characteristic $p$, over which all modules are assumed to be finite dimensional. Our notation resembles that of $[\mathbf{4}],[\mathbf{5}]: \mathbf{G}$ is a simply connected algebraic group (of simple type), $\mathfrak{g}$ its Lie algebra, $\mathscr{U}$ the restricted universal enveloping algebra of $\mathfrak{g}, G_{q}$ the group of rational points of $\mathbf{G}$ over a field of $q$ elements, $\mathscr{R}_{q}$ the group algebra of $G_{q}$ over $K$. (When $q=p$, we write simply $G, \mathscr{R}$.)

EXAMPLE. $\mathbf{G}=\mathbf{S L}(2, K), \mathfrak{g}=\mathfrak{s l}(2, K), G_{q}=\mathbf{S L}(2, q)$.

The set $\Lambda$ of restricted highest weights (determined by integers between 0 and $p-1$ ) indexes the (classes of) irreducible modules $M_{\lambda}$ for $\mathscr{U}$ (or $\mathscr{R}$ ). If $\lambda=\lambda_{0}+\lambda_{1} p+\cdots+\lambda_{k} p^{k}\left(\lambda_{i} \in \Lambda\right)$, then the twisted tensor product modules $M_{\lambda}=M_{\lambda_{0}} \otimes M_{\lambda_{1}}^{(p)} \otimes \cdots \otimes M_{\lambda_{k}}^{\left(p^{k}\right)}$ exhaust the (classes of) irreducible modules for $\mathscr{R}_{q}\left(q=p^{k+1}\right)$ and for $\mathbf{G}$ (as $k$ runs over all nonnegative integers). Denote by $U_{\lambda}, R_{\lambda}, R_{\lambda}$ the respective PIM of $\mathscr{U}, \mathscr{R}, \mathscr{R}_{q}$ having top composition factor $M_{\lambda}, M_{\lambda}, M_{\lambda}$. The only irreducible module which is also projective is the Steinberg module $M_{\sigma}=U_{\sigma}=R_{\sigma}, \sigma=(p-1) \delta$, $\delta=$ half-sum of positive roots. A similar statement is true for $M_{\sigma}=R_{\sigma}$ $\left(\sigma=\sigma+\sigma p+\cdots+\sigma p^{k}\right)$.

\section{Projective modules.}

Lemma. Let $V, W$ be modules for the restricted universal enveloping algebra of a restricted Lie algebra, with $W$ projective. Then $V \otimes W$ is also projective.

This is proved in [7]. The analogous statement for the group algebra of a finite group is well known [3, Exercise 2, p. 426].

We apply the lemma as follows. For $\mu \in \Lambda$, define $T_{\mu}=M_{\mu} \otimes M_{\sigma}$ ( $\sigma$ as above). This is a module for $\mathbf{G}, \mathscr{R}, \mathscr{U}$, and is projective for $\mathscr{R}, \mathscr{U}$ (since $M_{\sigma}$ is). In particular, $T_{\mu}$ is the direct sum of certain $\mathscr{U}$-modules $U_{\lambda}$.

AMS (MOS) subject classifications (1970). Primary 20C20; Secondary 20G40, 17B10.

Key words and phrases. Chevalley group, projective module, principal indecomposable module, modular representation theory, Cartan invariants, classical Lie algebra.

${ }^{1}$ Research supported in part by NSF Grant GP 28536. 
If $\mu \in \Lambda$, define its opposite $\mu^{0}$ to be $\tau_{0}(\mu+\delta)-\delta, \tau_{0}$ the unique element of the Weyl group which interchanges positive and negative roots. Our main result can now be formulated.

THEOREM A. Set $\lambda=(\mu-\delta)^{0}$. Then $U_{\lambda}$ occurs precisely once as a $\mathscr{U}$-summand of $T_{\mu}$ and is stable under $\mathbf{G}$, therefore is also a projective $\mathscr{R}$ module involving $R_{\lambda}$ as a summand. In particular, $\operatorname{dim} R_{\lambda} \leqq \operatorname{dim} U_{\lambda}$.

The proof uses some ideas from [4]. For $\mathbf{G}=\mathbf{S L}(2, K)$, a result of this type was first noticed empirically by the second author.

ReMARKs. (1) One can effectively compute (at least for small rank and small $p$ ) the modules $T_{\mu}$, starting with the known decomposition of tensor products of irreducible modules in characteristic 0 and then reducing modulo $p$. Using this approach and other data, the first author computed the Cartan invariants of SL $(3,5)$, avoiding Brauer's method.

(2) From the tensor product construction (and knowledge of the modules $M_{\mu}$ ) one also gets an effective, but lengthy, algorithm for computing the "decomposition" numbers $d_{\lambda \lambda}$ which figure in [4]. This in turn yields the Cartan invariants of $\mathscr{U}$.

Call $\lambda \in \Lambda$ regular if $\lambda=\sum m_{i} \lambda_{i}$ with all $m_{i}$ nonzero $\left(\lambda_{i} \in \Lambda\right.$ fundamental dominant weights). Empirical evidence, along with some heuristic arguments, suggests the following conjecture, which is true in rank 1 ([5], [6]) and also for $G=\mathbf{S L}(3,5), \mathbf{S L}(3,3), \mathbf{S p i n}(5,3)$.

Conjecture. As $\mathscr{R}$-modules, $U_{\lambda}=R_{\lambda}$ if and only if $\lambda$ is regular.

For the groups $G_{q}$, one obtains (as in [5], [6]):

THEOREM B. If $\lambda=\lambda_{0}+\lambda_{1} p+\cdots+\lambda_{k} p^{k}$, define $U_{\lambda}=U_{\lambda_{0}} \otimes U_{\lambda_{1}}^{(p)} \otimes$ $\cdots \otimes U_{\lambda_{k}}^{\left(p^{k}\right)}$ (as module for $\left.\mathbf{G}\right)$. Then $U_{\lambda}$ is a projective $\mathscr{R}_{q}$-module $\left(q=p^{k+1}\right)$, with $R_{\lambda}$ as a direct summand.

\section{REFERENCES}

1. A. Borel, Properties and linear representations of Chevalley groups, Sem. Algebraic Groups and Related Finite Groups, Lecture Notes in Math., vol. 131, Springer-Verlag, Berlin and New York, 1970. MR 41 \#3484.

2. C. W. Curtis, Representations of Lie algebras of classical type with applications to linear groups, J. Math. Mech. 9 (1960), 307-326. MR 22 \# 1634.

3. C. W. Curtis and I. Reiner, Representation theory of finite groups and associative algebras, Pure and Appl Math., vol. 11, Interscience, New York, 1962. MR 26 \# 2519.

4. J. E. Humphreys, Modular representations of classical Lie algebras and semisimple groups, J. Algebra 19 (1971), 51-79.

5., Projective modules for $\mathbf{S L}(2, q)$, J. Algebra (to appear).

6. A. V. Jeyakumar, Principal indecomposable representations for the group $\mathbf{S L}(2, q)$, J. Algebra (to appear).

7. B. Pareigis, Kohomologie von p-Lie-Algebren, Math. Z. 104 (1968), 281-336.

8. R. Steinberg, Representations of algebraic groups, Nagoya Math. J. 22 (1963), 33-56. MR $27 \# 5870$.

Courant Institute of Mathematical Sciences, New York University, New York, NEW YORK 10012

School of Mathematics, Tata Institute of Fundamental Research, Bombay, India 